\title{
Covid-19 and Reimbursement Mechanisms in Medical Economics; Retrospective(ffs) or Prospective(drg) Payment Healthcare Systems, Which One?
}

\author{
Nojomi $\mathrm{M}^{1}$, Banihashemi $\mathrm{AT}^{2}$ and Ahangar $\mathrm{A}^{3 *}$ \\ ${ }^{1}$ Department of community and family medicine, Preventive Medicine and Public Health Research Center \\ ${ }^{2}$ Department of Community and Family Medicine, School of Medicine, Psychosocial Health Research Institute Assistant Professor of community and \\ preventive medicine, department of community and family medicine, Preventive Medicine and public Health Research Center (PMPHRC) \\ ${ }^{3}$ PhD in Health Economics, Postdoctoral Fellowship, Preventive Medicine and Public Health Research Center, Department of Community Medicine, School \\ of Medicine, Psychosocial Health Research Institute, Iran University of Medical Sciences, Tehran, Iran. \\ *Corresponding author: Ahangar A, PhD in Health Economics, Postdoctoral Fellowship, Preventive Medicine and Public Health \\ Research Center, Department of Community Medicine, School of Medicine, Psychosocial Health Research Institute, Iran University \\ of Medical Sciences, Tehran, Iran.
}

To Cite This Article: Nojomi M, Banihashemi AT, Ahangar A. Covid-19 and Reimbursement Mechanisms in Medical Economics; Retrospective(ffs) or Prospective(drg) Payment Healthcare Systems, Which One?. Am J Biomed Sci \& Res. 2021 - 14(2). AJBSR.MS.ID.001973. DOI: 10.34297/AJBSR.2021.14.001973.

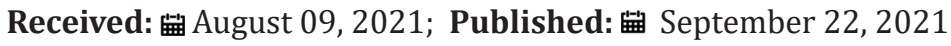

\section{Dear Editor}

Over the past two years, the COVID-19 pandemic has plagued hospitals with rapid and unexpected changes in all aspects, especially funding and financing, revenue generation, and healthcare reimbursement mechanisms. Health system policymakers and hospital senior managers oversee the provision of resources such as financial, human, equipment and devices to meet the needs of the people during the COVID-19 crisis. This monitoring in various dimensions of economic operation of hospital beds, creating new capacity of beds. Review of key hospital processes and the resilience threshold of hospitals in the face of crises. outbreaks COVID-19 has been a lot of volatility, but the trend spreads rapidly around the world, not only not less but in recent months has been even greater. The prevalence of COVID-19 depends on various factors such as health infrastructure, health, economic, social, and cultural policies of countries and varies from country to country. The fluctuations of the spread of this disease in the form of different waves (first, second, third, and fourth waves, etc.) have appeared in many countries of the world, including Iran (in the fifth wave stage). One of the biggest concerns about COVID-19 is the financial and economic effects of the disease on health financing and reimbursement systems. During the period of increasing COVID-19 disease, hospitals made these changes to meet the increasing demand for COVID-19, while a large proportion of non-COVID patients for various reasons from hospitalization for elective hospitalization such as inpatient Suspended operation (OR). This issue is especially important for hospitals, as the most important components and beating heart of any health system. Today, disease (such as COVID-19 pandemic disease), the opposite of health, is one of the most important health problems of health systems and in a way one of the biggest economic, political, and social problems in the world for all countries with any level of income (GDP). This challenge is higher than average and even lower for middle-income developed countries, while it is more acute for low-income developing countries, which means that in low-income countries, patients have to pay more for to treat, and this puts many people below the poverty line and worsens the status of the "catastrophic health costs" index. COVID-19 disease has economic and financial effects at both macro and micro levels. At the macro level, the economic effects of this pandemic disease have emerged as a shock or economic crisis, meaning that it has affected the financing and economic structure of countries, in other words, the economic pattern and turnover worldwide. At the micro-level, funding sources and health care reimbursement mechanisms in hospitals have been severely affected. In this study, health care repayment and financing systems in the health sector, especially hospitals, were studied. In addition, are retrospective health financing and repayment systems better or prospective repayment systems in times of crisis or economic shock caused by COVID-19 disease?

The types of financing mechanisms and current repayment methods, depending on the type of health system as well as other conditions and requirements of each country, have strengths and weaknesses [1-3]. The results of a study (Ahangar et al., 2021) at Iran University of Medical Sciences show that before and after COVID-19 disease, hospitals with prospective repayment systems (such as DRG, Global, Per Capita mechanisms) compared to hospitals with retrospective repayment systems were more efficient in controlling costs and resources, as well as better managing the quality of hospital care.

Keywords: COVID-19, Hospital, Reimbursement mechanisms, Retrospective, Prospective, Healthcare financing, Hoapital economics 


\section{Introduction}

Today, the health sector is one of the most important service sectors and health status is one of the indicators of development and social welfare, so economic recognition and a kind of financial re-engineering of this sector is important in the COVID-19 Period. Health systems are one of the largest sectors of the world economy. Global spending on health care accounts for approximately 8\% of GDP. In most developing countries, about 5 to 10 percent of government spending goes to the health sector [1-3].

Health is one of the most important issues for governments in the field of public services and welfare, and its establishment at the macro-level of society depends on the adoption of policies by policymakers and health officials. Meanwhile, health financing policies and payment systems have a very important effect on the efficiency of the health system and the optimal control of costs. The effects of financing systems and various health care repayment mechanisms and related challenges on increasing or decreasing the costs of the health sector and hospitals in times of crisis, such as the COVID-19 pandemic, are significant. It is clear that in different health systems, none of the mechanisms for receiving and paying health costs is complete and flawless, and each will somehow direct financial risk to the recipients or service providers. Therefore, health care providers are expected to choose a combination of the best options in order to cover as many services and efficiency as possible, the most important tools in the health system, according to the socio-economic conditions. It is obvious that efficient and effective payment and payment systems in society should consider the rights of all parties in a reasonable and fair manner. What the current system of the country shows is the high percentage of the people's share in providing health expenses, which should be addressed by adopting appropriate policies [2-3]. One of the most immediate and important challenges for many developing and underdeveloped (low-income) countries is the provision of health care mechanisms for the more than 1.5 billion poor living on the surface. Worldwide, more than 80 percent of the total health expenditures of low-income countries are related to out-of-pocket (OOP) payments. High OOP index, high costs of medical care, and diagnostic treatment are the main reasons for catastrophic health costs (CHE) as well as poor health costs; (Limited financial resources have posed various challenges to most of the world's health systems in terms of service quality, efficiency and effectiveness, and justice [1-3].

Health, as a necessary commodity is very sensitive, because insufficient attention to this category can, in addition to the damage to the health of society, cause the huge resources that are spent in this sector to be wasted. One of the important goals that all countries pursue in their reforms in the health sector is cost control, which, of course, along with other goals such as increasing efficiency and effectiveness in the health system and ultimately improving the quality of services. In the literature of health financing, there are three main and definite issues: first, that the cost of health services is increasing day by day, and second, that science and technology are constantly evolving without stopping. Third, the demographic picture of the world is on the rise [4-6]. Today, having a more successful financial balance is one of the main and key criteria for the success of any organization or manufacturing or service institution such as hospitals and insurance companies. Hospitals as a subset of the health care system, regardless of the fact that the services they provide related to human life, but the need for proper management of resources, including physical, financial, and human resources to minimize costs and optimal and effective use of resources in Existence is inevitable [1,3,7].

One of the most important control nobs in the health system is financing systems and repayment mechanisms. All organizations that mobilize and circulate funds for the health sector must decide who is to be paid, why, and how much. One of the most important ways to control treatment costs in different health systems is to use proper payment systems in the health sector [8]. Reimbursement mechanisms for healthcare have used a variety of payment mechanisms with varying degrees of effectiveness. Whether these mechanisms are used singly or in combination, it is imperative that the resulting systems remunerate on the basis of the quantity, complexity, and quality of care provided. Expanding the role of the reimbursement and health financing structure to monitor provider practice, patient responsiveness, and functioning of the healthcare organization has the potential to not only enhance the accuracy and efficiency of reimbursement mechanisms but also to improve the quality of medical care [9-11].

Care payment mechanisms should be designed and implemented in a way that allows service providers to attract enough revenue so that they are sufficiently motivated to give high quality services and prevent them from moving to more lucrative jobs [11].

Studies show that the prevalence of COVID-19 pandemic disease has significant effects on the financing structure and health economy in several ways: [9-11] These effects are divided into three categories: short-term, medium-term, and long-term. Short-term effects: COVID-19, on the one hand, due to the nature of its acute respiratory infection, causes an increasing trend in the cost of health care, drugs, respiratory equipment, and other preclinical diagnostic and therapeutic services related to COVID-19, subsequently, increasing Attention is paid to total health expenditure. Second, the medium-term effects; The recession caused by the disease, on the one hand, affects future health expenditures through reduced employment (increased unemployment), declining GDP, as well as declining tax revenues and declining GDP. Becomes. On the other 
hand, the disease delays other non-COVID-19 -related health care (such as elective surgery), which in turn creates an extra financial burden on future health care budgets. Third, long-term effects; The costs of rehabilitating the physical and mental health caused by the disease (and the resulting recession) will continue even after the COVID-19 incidence rate is eliminated or remain constant in the coming years, putting continued pressure on national health budgets for It will have years to come [10-11].

COVID-19 has affected the performance and economy of hospitals in several ways. The first is from the perspective of the recipient of services and care, the second is from the perspective of the provider of care and diagnostic services (medical and nursing team, hospital). From the first perspective, recipients of health care are two groups of outpatients and inpatients who are referred to the hospital for COVID-19 and other non-COVID-19 diseases. Since the outbreak of COVID-19, physician visits have declined significantly, due in part to the cancellation or postponement of scheduled visits for surgery by patients (public fear of -COVID 19) or hospital (Allocation of inpatient beds, specialized medical and nursing teams for outpatient visits and providing inpatient care and other diagnostic and therapeutic services for patients with or suspected of having COVID -19. It has also reduced activity time and in some cases even disabled hospital operating rooms, especially elective (non-emergency) surgeries [10-12].

One of the most important components of a hospital economy is healthcare reimbursement systems [2]. health care reimbursement mechanisms in health systems include methods such as Global Fee for Services (FFS), Per capita, Salary, Performance Pay (P4P), and Diagnostic Payments (DRGs, Diagnostic Related Groups). Health reimbursement mechanisms vary from country to country, which means that some countries may include a combination of all of these mechanisms, or some countries may have one or two of these reimbursement mechanisms. But the important point is that each of these repayment mechanisms has been created for various reasons such as globalization, having strengths or weaknesses, enforceability and operationalization according to specific social, economic, and political situations. Studies show that these mechanisms in health systems reflect different dimensions of the quantity, complexity, and quality of health care in varying degrees if health reimbursement mechanisms are combined and updated with other technologies such as HIS, HIT. In this case, the efficiency and performance of each of these mechanisms or a combination of these mechanisms will be better [13-14].

Repayment methods in the health system are various and in the country, based on the needs and facilities, one or a set of several methods are used to compensate for services. The effects and incentives of the payment system, both in terms of demand and supply, are to some extent dependent on an effect called ethical risk. In terms of supply, the payment system gives rise to a complex response from physicians and hospitals. The impact of payment on the volume of care occurs because physicians can induce demand for their services [15]. The types of financing mechanisms and current repayment methods, depending on the type of health system as well as other conditions and requirements of each country, have strengths and weaknesses. According to studies, health care reimbursement systems are divided into two general categories, retrospective, and prospective reimbursement systems. In retrospective reimbursement systems, the service provider is unaware of the exact amount of money it will receive and usually receives a fee shortly after the service is provided. The following methods are retrospective, such as FFS(Fee-For-Services) and pay for a day. In prospective reimbursement systems, the provider is not only aware of the amount received, but in some cases even receives the cost before the service is provided, and in fact, the service provided by him is pre-purchased. Prospective reimbursement systems are such as GLOBAL Budget, DRG (Diagnosis Related Groups), Pay per Capita [16-19].

In the world, health systems have different health financing structures and hospital reimbursement systems. This means that different countries have different health systems based on economic, political, social and cultural requirements, and consequently different financing structures for the health and hospital sectors. Accordingly, several key questions arise, first, whether health systems are equally effective or not? Second, what are the criteria for the efficiency or inefficiency of different health systems? Can a comparison of inputs (health financing structure and reimbursement systems) or health outputs be the basis for evaluating the effectiveness of health systems? Are health financing systems effective in times of economic crisis or disease such as the COVID-19 pandemic? Which one or combination of health care reimbursement systems is effective in the COVID-19 pandemic? Answering these questions, the need to discuss key concepts such as "health financing", "risk accumulation", "risk sharing", "health care repayment systems", retrospective repayment mechanism, prospective repayment mechanism "in the economic literature Explains the health and economy of the hospital more than ever [18-20].

\section{Method}

The present study is a cross-sectional and applied research. In this study, first all the cost items of service procedures and length of hospital stay recorded in the files were extracted from the hospital information system, then the average cost and stay of the patient for each surgery was determined and the cost and average 
stay of the patient determined in the future repayment system. Perspective (Global, etc.) and retrospective (Fee-For-Services) of Iran University of Medical Sciences in 2019 were compared. Finally, there is a significant difference in costs between prospective and retrospective repayment systems, average cost with each of the effective factors such as average length of stay, type of insurance, type of ward, age of patients after examining the assumption of normalcy. Data distribution and using t-test, Mann-Whitney, analysis of variance, Kruskal-Wallis and linear regression methods were evaluated using SPSS. 21 software.

\section{Results}

About 2 years after the outbreak of COVID-19 pandemic, COVID-19 has economic and financial effects at both macro and micro levels. At the macro level, the economic effects of this pandemic disease have emerged as a shock or economic crisis, which means that it has affected the financing and economic structure of countries, in other words, the economic model and turnover in the world. At the micro level, funding sources and health care reimbursement mechanisms in hospitals have been severely affected. At the macro level, COVID-19 has caused a variety of economic crises in countries, including the first type, the V-shaped economic shock (Canada in 2008) or the "classic shock" in which economic growth is first gradually declining, then Significantly returns to the previous economic situation (gradual upward trend). The second type is the U-shaped economic shock (as in the United States in 2008), in which economic growth declines sharply (falls) and subsequently improves slowly, but never returns to preeconomic shock. U-shaped As the recession escalates, this does more damage to labor productivity and productivity. The third type is the L-shaped economic shock (such as Greece, Iran) (in this type, economic growth is slow but economic growth is never Does not improve). In general, the worst type of economic shock is L-shaped and then U-shaped economic shock [19-20].

According to economists, the main determinant of the economic shock caused by COVID-19 is the ability of the shock to damage the supply and demand pattern of the economy (mainly the supply side of the economy) and, more specifically, on investment and GDP. Is. This means that with the onset of COVID-19 economic shock, first, credit intermediation factors (between stakeholders such as input suppliers, producers, distributors, and ultimately consumers) are disrupted., Consequently, capital stocks do not grow, economic growth slows (and even stagnates), and this leads to unemployment (dismissal) of workers from the workforce, skills are lost, productivity is reduced, and ultimately leads to Shock occurs systematically or structurally $[20,21]$.

According to various studies, health and its components (health financing structures) by economic growth and social development of communities and vice versa, economic growth is also affected by the health status of communities. In recent years, countries with higher economic growth are more likely to have more coherent health financing structures. This means that with the improvement and economic growth of countries, the percentage of GDP allocated to the health sector increases and, so, the probability that the state of health indicators will improve, as well as improving the health of society, ie having healthy and productive human capital, Increase GDP, and subsequently reach and socio-economic development of societies [22]. On the other hand, with the pandemic outbreak of COVID-19 over the past two years, it has slowed economic growth and even recession in countries reduced gross domestic product (GDP), and subsequently affected health care financing and repayment systems. While increasing total health expenditures, it affects health status (health outputs) such as mortality rates, fertility, and productivity, life expectancy, etc $[3,9,23]$.

According to WHO data (2020) over the past decades, economic development was positively associated with total health spending and a shift away from a reliance on out-of-pocket (OOP) towards government spending. The largest health spending growth rates were in lower-middle-income groups (5.0\%), in low-income countries (grew nearly as fast, at $4.6 \%$ ). In 2018, in the world outof-pocket (OOP) was $18.18 \%$ of total health spending (from $19.39 \%$ in 2000 to $18.12 \%$ in 2018 ) and in low-income and lower-middleincome countries, $29 \cdot 1 \%$ of all health spending was financed by the government, and $58.0 \%$ of spending was OOP spending and $12.9 \%$ of spending was development assistance $[3,9,22]$. Also, it is estimated that by 2040, the growth of health expenditure in government spending as a share of the total for Africa (39.0\%), in Out-of-pocket spending (31.1\%) and in development assistance for health for Africa (14.4\%) [22].

A crucial concept in health financing is that of risk-pooling, and the larger the degree of pooling, the fewer people will have to bear the financial consequences of their own health risks. Health financing systems encompass various degrees of risk-sharing. According to the method recommended by WHO, there are three categories of risk-sharing: a) low-risk sharing, b) medium risksharing and c) advanced risk-sharing (5). There are essentially four classes of approach to risk-pooling; (no risk pool, unitary risk pool, fragmented risk pools, integrated risk pools), under which fragmented risk pools are compensated for the variations in risk to which they are exposed [3,10,23].

WHO's data (Ahangar et al, 2020) showed that between 20002020 the degree of risk-sharing in health financing systems are various, for example in Africa (from1.68 to 2.62; of the total 6 points Likert) is low risk-sharing, This slow shift in this region was coincided with increasing to share of public expenditure of GDP and of total expenditures, in Africa (41.95 to 46.84), and also with 
reducing share in the private expenditure of total; expenditures particularly Out-Of-Pocket, in Africa (45.58 to 39.01) [3,10,23].

Also, the results of this study showed that the group of countries with higher incomes have a higher degree of risk-sharing (health financing). That is, by increasing the income of countries and moving from the group of low-income countries to the group of middle-income countries (lower and higher than average) and then the group of high-income countries, the average degree of health financing risk-sharing among groups Different incomes increase significantly. In addition, such an increasing trend is observed in each of the different income groups. As the group of low-income countries has the lowest average degree of financing risk sharing (average 1.88, changes from 1.57 in 2000 to 2.08 in 2020), the group of countries with lower than average income has the average degree of sharing Risk 2.66 (with changes of 0.40 from 2000 to 2020), group of countries with above-average income with an average degree of risk-sharing of 3.79 (with changes of 0.48 during 2000 to 2020), group of high-income countries with an average degree of risk-sharing of 5.18 ( With changes of 0.92 from 2000 to 2020). In Iran, the average degree of risk sharing in health financing is 3.02 and incremental changes are 1.10 (from 2.50 in 2000 to 3.60 in 2020), in the EMRO region the average of the index is 3.51 and incremental changes are 0.70. (From 3.20 in 2000 to 3.90 in 2020), and in the world it is 3.96 and incremental changes are 0.83 (from 3.52 in 2000 to 4.35 in 2020$)[3,7,8,21,23]$.

Studies showed that health systems with a publicly funded health financing structure (such as public government resources, tax revenues, social insurance prepayments, and private insurance) are more efficient in achieving public health outputs in countries, health financing structures have better risk-sharing indexes, especially in economic crises and pandemic diseases such as COVID-19. Conversely, countries with a health financing structure based on out-of-pocket payments, deductibles, etc., have lower risk accumulation and sharing, and consequently during the COVID-19 period, with increasing direct and indirect health care costs. It imposes an additional financial burden on individuals and households $[3,7,8,15]$.

Risk-sharing in health financing is defined as "Any system which allows payors to share some of the financial risk associated with a particular patient population with providers. Providers agree to adhere to fixed fee schedules in exchange for an increase in their payor base and a chance to benefit from cost containment measures. Common risk-sharing methods are prospective payment schedules (PROSPECTIVE PAYMENT SYSTEM), capitation (CAPITATION FEES), diagnosis-related fees (DIAGNOSIS-RELATED GROUPS), and pre-negotiated fees" [24].

Several studies (such as Ahangar et al. 2021, Ikegami 2009, Ikegami 2015) [13-15] have argued that each of the health care financing and repayment mechanisms have different dimensions of "cost control", "impact on quality", "executive management" and por are negative. Prospective payment mechanisms such as (DRG, Case-mix, per case) as well as Fee-for-Services payment mechanism in terms of executive management, this type of payment system are one of the most difficult health care payment mechanisms. Other repayment mechanisms such as per capita payment, daily fee payment is very easy in terms of executive management, and reward repayment mechanisms, fixed-rate payment, salary payment and budget payment is relatively easy in this regard. Studies also show that the Fee-for-Services mechanism is very week in terms of the "cost control" criterion among all types of reimbursement mechanisms, while other reimbursement mechanisms are good in terms of this criterion (occasional fixed-rate payments, payment rewards, fixed-rate payments), relatively good (payroll, daily fee payment) and very good (DRG, per capita payment, budget payment, global). Also, the types of health care reimbursement mechanisms can be evaluated in terms of "impact on quality" criteria. Accordingly, studies have shown that some repayment mechanisms have a very good impact on the quality of care, including occasional payments (DRG, Case-mix, per case), good (bonus payments, fixedrate payments), relatively good (per capita payment, payment Budget) while the mechanisms for reimbursement of daily fees, wages, and salaries are weak in terms of quality impact criteria. The important point is that the repayment mechanism has an effect on good quality in good competitive conditions, but the same repayment mechanism, if not in competitive conditions, and may lead to the induced demand phenomenon (SID), is poor in terms of quality criteria [25-27].

\section{Discussion}

During COVID-19, one of the biggest problems for hospitals is the problem of hospital economics and the challenge of resource constraints. The challenge of severely limited resources in the face of unlimited needs and the demand of patients in hospitals Many problems, including the large number of patients, poor quality of services, lack of diagnostic tools and equipment, worn and worn devices and equipment, long queues in clinics, and provides outpatient operating rooms, shortages of medicine and other medical equipment, shortages of medical and nursing staff, and other support staff. Health economists, three main challenges in the field of hospital economic management (especially the COVID-19 pandemic period): 1) the challenge of resource supply and production, 2) the challenge of optimal resource management, and 3) the challenge of resource allocation. The main economic challenge of resource allocation in hospitals, especially during the COVID-19 pandemic period, is production and cost function. These two functions are related to the challenge of hospital efficiency and effectiveness. 
Understanding the structure of financing and costs of medical procedures and services, including inpatient/outpatient, has an important role in rational cost management and optimal use of resources in achieving the quality and effectiveness of medical services. In fact, reimbursement mechanisms are one of the most important factors influencing the cost of hospital services and surgeries. In the meantime, reimbursement mechanisms have been the most important financial management tool in the hospital so that the proper implementation and deployment of prospective payment systems (such as global, DRG) can be used as a suitable tool for cost control and resource management. Used in hospitals. Awareness of the financial burden-sharing of effective factors in providing hospital services and procedures, especially global service packages, ultimately lead to optimal resource management, cost control, and rationalization, as well as providing better quality care to patients. In particular, the LOS (Length of Stay) index, the difference between better days and the standard set in the future global repayment system compared to the retrospective Fee-ForServices has decreased in most cases and the difference between the average hospital days has been significant. Therefore, according to the above cases, it is necessary to review the payment system, set up a prospective repayment system based on DRG and Case-Mix disease coding systems and implement a futuristic payment system in the country.

Hospital reimbursement systems are one of the most important components of hospital economics in the efficiency and performance of hospitals during the COVID-19. The types of financing mechanisms and current repayment methods, depending on the type of health system as well as other conditions and requirements of each country, have strengths and weaknesses. The results of a study (Ahangar et al., 2021) at Iran University of Medical Sciences show that before and after COVID-19 disease, hospitals with prospective repayment systems (such as DRG, Global, Per Capita mechanisms) compared to hospitals with retrospective repayment systems were more efficient in controlling costs and resources, as well as better managing the quality of hospital care.

\section{Financial Support and Sponsorship}

This study was conducted with the financial support and assistance of Iran University of Medical Sciences, Research Center for Preventive Medicine and Population Health.

\section{Acknowledgment}

The authors declare that there is no conflict of interests.

\section{References}

1. Iswanto H (2018) Hospital Economics:A Primer on Resource Allocation to Improve Productivity \& Sustainability. Taylor and Francis
2. Ali A, Mohammad AA, Hossein MA, Sajjad FD, Samaneh S (2019) Transition of Health Financing, Cost Risk-Sharing and Risk-Pooling Models in the Health Sector: A Systematic Review (1990-2019). IRCMJ 21(12).

3. (2000) World Health Organization. The World Health Report 2000 Health Systems: Improving Performance. World Health Organization, Geneva.

4. (1999) WHO. Economic Dimensions of Health care (With Special Reference to Health Insurance). Geneva: WHO.

5. Mohagheghi MA (2004) Review the Situation and Prescribe Antibiotics in Tehran City Between the Years 1998 till 2001. The Final Report of the Research Project. Tehran.

6. Ahangar A, Ahmadi AM, Mozayani AH, Faraji Dizaji S (2018) The Role of Risk-sharing Mechanisms in Finance Health Care and Towards Universal Health Coverage in Low-and Middle-income Countries of World Health Organization Regions. Journal of Preventive Medicine and Public Health51(1): 59-61.

7. Ahangar A, Ahmadi AM, Mozayani AH, Faraji Dizaji S (2018) Why are risk-pooling and risk-sharing arrangements necessary for financing healthcare and improving health outcomes in low and lower middleincome countries?. Health Journal 10(1): 122-131.

8. Britton JR (2015) Healthcare Reimbursement and Quality Improvement: Integration Using the Electronic Medical Record. Int J Health Policy Manag 4(8): 549-551.

9. (2020) World Health Organization. Novel coronavirus (2019-nCoV) situation reports. Geneva: World Health Organization.

10. Ornell F, Schuch JB, Sordi AO, Kessler FHP (2020) "Pandemic fear" and COVID-19: mental health burden and strategies. Braz J Psychiatry 42(3): 232-235.

11. Shin J h, Takada D, Morishita T, Lin H, Bun S, et al. (2020) Economic impact of the first wave of the COVID-19 pandemic on acute care hospitals in Japan. PLoS ONE 15(12): e0244852.

12. Pourreza A (2005) Health Economics for Developing Countries. Tehran:Institude for Management and Planing Studies. 73-107

13. Flodgren G, Eccles MP, Shepperd S, Scott A, Parmelli E, et al (2011) An overview of reviews evaluating the effectiveness of financial incentives in changing healthcare professional behaviors and patient outcomes. Cochrane Database Syst Rev 2011(7)

14. Ahangar A, Nojomi M, Tehrani Banihashemi A (2021) Economic evaluation and comparative study of factors affecting surgical costs in DRGs / Case mix), (Global and Retrospective Repayment System (FFS: Fee-For-Service)) in Iran University of Medical Sciences, (the COVID-19 period) . Postdoctoral fellowship. Dissertation.

15. Ikegami N (2015) Fee-for-service payment - an evil practice that must be stamped out?. Int J Health Policy Manag 4(2): 57-59.

16. Ikegami N (2009) Games policy makers and providers play: Introducing case-mix-based payment to hospital chronic care units in Japan. J Health Polit Policy Law 34: 361-380.

17. Szlezak PC, Reeves M, Paul Swartz P (2020) Understanding the Economic Shock of Coronavirus. Harv Bus Rev.

18. Yoonyoung C, Jorge A, Yasuhiro K, Douglas J, Ruth R (2021) The Impact of the COVID-19 Pandemic on Low Income Households in the Philippines: Impending Human Capital Crisis. COVID-19 Low Income HOPE Survey.

19. Sharma R (2018) Health and economic growth: Evidence from dynamic panel data of 143 years. PLOS ONE 13(10): e0204940. 
20. Ahangar A, Ahmadi AM, Mozayani AH, Faraji Dizaji S (2018) The Assessment of Risk-Sharing in the Financing of the Health Sector and Health Outcomes; a Comparative Study across WHO's Regions. PhD. Dissertation, Tarbiat Modarres University (TMU) Tehran.

21. Dieleman JL Templin T, Sadat N, Reidy P, Chapin A, et al (2016) National spending on health by source for 184 countries between 2013 and 2040 . The Lancet. 387(10037): 2521-2535.

22. Ahangar A, Ahmadi A, Mozayani A, Faraji Dizaji S (2018) Financing for Healthcare in Sub-Saharan Africa and South Asia: Risk Pooling \& RiskSharing Arrangements; Essential Strategy for Overcoming Poverty Costs . Iran J Health Sci 6(2): 64-65.

23. (2015) World Health Organization. Regional Office for the Western Pacific. Case-based payment systems for hospital funding in Asia: an investigation of current status and future directions. Manila: WHO Regional Office for the Western Pacific.
24. Anderson G (2009). The effects of payment by results. British Medical Journal 339.

25. Appleby J, Harrison T, Hawkins L (2012) Payment by Results: How Can Payment Systems Help to Deliver Better Care? The King's Fund.

26. Busse R, Geissler A, Quentin W (2011). Diagnosis-Related Groups in Europe: moving towards transparency, efficiency and quality in hospitals. McGraw-Hill International.

27. Busse R, Stahl J (2014) Integrated care experiences and outcomes in Germany and the Netherlands. Health Affairs 33(9): 1549-1558. 